Research Paper

\title{
Determination of risk factors for predicting the onset of symptoms in asymptomatic COVID-19 infected patients
}

\author{
Pei-Yao $\mathrm{Tao}^{1^{*}}$, Ling Leng ${ }^{2 *}$, Kun Liu ${ }^{3}$, Ri-Hua Zhou ${ }^{4}$, Yue-Chun $\mathrm{Hu}^{5}$, Shang-Jie $\mathrm{Wu}^{6}$, Yu-Dong Xiao ${ }^{\circledR}$ and \\ Jun Liu ${ }^{\boxplus}$ \\ 1. Department of Radiology, The Second Xiangya Hospital, Central South University, Changsha, China, 410011. \\ 2. Department of Cell Biology, School of Basic Medical Science, Tianjin Medical University, Tianjing, China, 300070. \\ 3. Department of Infection and rehabilitation, Yiyang The Fourth People's Hospital, Yiyang, China, 413000 \\ 4. Medical Department, Chenzhou The Second People's Hospital, Chenzhou, China, 423000. \\ 5. Department of Radiology, Loudi Central Hospital, Nanhua University, Loudi, China, 417000. \\ 6. Department of Pulmonary and Critical Care Medicine, The Second Xiangya Hospital, Central South University, Changsha, China, 410011. \\ *These authors contributed equally to this study.
}

$\square$ Corresponding authors: Yu-Dong Xiao, Department of Radiology, The Second Xiangya Hospital, Central South University, No.139 Middle Renmin Road, Changsha, Hunan, 410011, China. Phone: +86-13637403027; Fax: +86-13637403027; E-mail: xiaoyudong222@csu.edu.cn; Jun Liu, Department of Radiology, The Second Xiangya Hospital, Central South University, No.139 Middle Renmin Road, Changsha, Hunan, 410011, China. Phone: +86-13787085002; Fax: +86-13787085002; E-mail: junliu123@csu.edu.cn.

(c) The author(s). This is an open access article distributed under the terms of the Creative Commons Attribution License (https://creativecommons.org/licenses/by/4.0/). See http://ivyspring.com/terms for full terms and conditions.

Received: 2020.04.29; Accepted: 2020.07.26; Published: 2020.08.06

\begin{abstract}
Background: The number of asymptomatic infected patients with coronavirus disease 2019 (COVID2019) is rampaging around the world but limited information aimed on risk factors of asymptomatic infections. The purpose of this study is to investigate the risk factors of symptoms onset and clinical features in asymptomatic COVID-19 infected patients.

Methods: A retrospective study was performed in 70 asymptomatic COVID-2019 infected patients confirmed by nucleic acid tests in Hunan province, China between 28 January 2020 and 18 February, 2020. The epidemiological, clinical features and laboratory data were reviewed and analyzed. Presence or absence at the onset of symptoms was taken as the outcome. A Cox regression model was performed to evaluate the potential predictors of the onset of symptoms.

Results: The study included 36 males and 34 females with a mean age of $33.24 \pm 20.40$ years (range, 0.5-84 years). There were 22 asymptomatic carriers developed symptoms during hospitalization isolated observation, and diagnosed as confirmed cases, while 48 cases remained asymptomatic throughout the course of disease. Of 70 asymptomatic patients, 14 (14/70, 20\%) had underlying diseases, 3 (3/70, 4.3\%) had drinking history, and 11 (11/70, 15.7\%) had smoking history. 22 patients developed symptoms onset of fever $(4 / 22,18.2 \%)$, cough $(13 / 22,59.1 \%)$, chest discomfort $(2 / 22,9.1 \%)$, fatigue $(1 / 22,4.5 \%)$, pharyngalgia $(1 / 22,4.5 \%)$ during hospitalization; only one $(1 / 22,4.5 \%)$ patient developed signs of both cough and pharyngalgia. Abnormalities on chest CT were detected among 35 of the 69 patients (50.7\%) after admission, except for one pregnant woman had not been examined. 4 (4/70, 5.7\%) and 8 (8/70, $11.4 \%$ ) cases showed leucopenia and lymphopenia. With the effective antiviral treatment, all the 70 asymptomatic infections had been discharged, none cases developed severe pneumonia, admission to intensive care unit, or died. The mean time from nucleic acid positive to negative was $13.2 \pm 6.84$ days. Cox regression analysis showed that smoking history $(\mathrm{P}=0.028$, hazard ratio $=4.49,95 \% \mathrm{Cl} 1.18-17.08)$ and existence of pulmonary disease $(P=0.038$, hazard ratio $=7.09,95 \% \mathrm{Cl} 1.12-44.90)$ were risk factors of the onset of symptoms in asymptomatic carries.
\end{abstract}

Conclusion: The initially asymptomatic patients can develop mild symptoms and have a good prognosis. History of smoking and pulmonary disease was prone to illness onset in asymptomatic patients, and it is necessary to be highly vigilant to those patients.

Key words: COVID-19; asymptomatic infected patients; symptoms onset; risk factors; predictive value 


\section{Introduction}

Since December 2019, with the emergence and expansion of coronavirus disease 2019 (COVID-2019) or the severe acute respiratory syndrome corona virus 2 (SARS-CoV-2), the World Health Organization (WHO) has declared COVID-19 as the sixth public health emergency of international significance on 30 January 2020 [1,2]. As of 10 April 2020 a confirmed more than one million five hundred and ninety thousand people have been infected with COVID2019 globally, among them, 353807 cases reported cured and 95495 cases reported deaths. After several months of containment, China has transitioned to a mitigation phase. However, the clinical manifestations of COVID-19 are protean and can present as an asymptomatic carrier state. Asymptomatic COVID-2019 infected patients were defined as follows [3-5]: patients who tested (a) positive result of reverse transcription polymerase chain reaction (RTPCR) testing for nasal or pharyngeal swab specimens twice every 24 hours; (b) without any conscious clinical symptoms prior to diagnosis, such as fever, cough, fatigue, sore throat, muscle pain, etc., and (c) the presence or absence of pulmonary pathological changes on the chest computed tomography (CT) examination after the diagnosis of infection. Early recognition of infections and cutting off the route of transmission are key points to control COVID-19. However, it is precisely because of asymptomatic and poor prevention awareness that such patients may not be given full attention and bears several challenging problems, including insidious symptom onset, subclinical manifestations and the undetectability during early stage of infection. To date, accumulated evidence has revealed that the asymptomatic carriers are infectious to an extent and can transmit COVID-19 via person-to-person contact [2,4-7]. Zou, et al. [8] reported that the viral load detected in asymptomatic patients was comparable to that found in symptomatic patients, which suggested that transmission potential in asymptomatic patients was not low. Therefore, it is crucial to identify and isolate asymptomatic carriers in order to contain the outbreaks in advanced stages. However, limited data aimed on risk factors are available for asymptomatic infections. The purpose of this study is to present the risk factors of symptoms onset and clinical features in asymptomatic infected patients.

\section{Material and method}

\section{Study population}

This study was subject to approval by our Medical Ethical Committee (Approval No. 2020002), which waived the requirement for patients' informed consent referring to the Council for International Organization of Medical Sciences (CIOMS) guideline.

We retrospectively retrieved data for a total of 70 laboratory-confirmed positive asymptomatic carriers with COVID-19 between 28 January 2020 and 18 February 2020 by the nucleic acid in Hunan province, China. The diagnosis of COVID-19 infection based on the guidelines of China National Health Commission: Diagnosis and treatment of pneumonitis caused by new coronavirus (trial version 6) [8]. Asymptomatic infections were found and quarantined in the following ways: (a) close contact with the confirmed COVID-2019 patients within 14 days; (b) with a history of travel to Wuhan or residence in epidemic areas; (c) cluster epidemic investigation; and (d) exposed population during infectious source tracking.

\section{Data collection}

We gathered epidemiological and clinical features, laboratory examination and outcome of disease from individuals with 70 asymptomatic COVID-19 patients, via inpatient medical records in 10 designated hospitals in Hunan province, China. Basic information and demographic characteristics were collected as the following: name, age, gender, dates of diagnosis, dates of symptoms onset, and dates of each chest CT examination. Clinical information on each case during their hospitalization was also recorded the underlying disease history, smoking and drinking history, the first onset of symptoms during quarantined and the corresponding dates. The definition of positive chest $\mathrm{CT}$ imaging findings was that the patients were presence of the CT imaging features that favored viral pneumonia such as ground-glass opacity (GGO), bilateral patchy shadowing, local patchy shadowing, as well as interstitial abnormalities [8]. Laboratory tests were conducted after admission, including leukocyte count (WBC), lymphocyte count (LY), neutrophil ratio (NUET), and lymphocyte ratio (LYM). The retrospective study began with the diagnosis of asymptomatic infection with COVID-2019, and absence or presence of symptoms was taken as the outcome. For patients with symptoms of onset, the follow-up time was the interval between the date of symptoms onset and the date of nucleic acid positive, while for remained asymptomatic carriers was the time interval between nucleic acid positive and negative. Fitness for discharge was based on the absence of obvious symptoms, with improved evidence on chest $\mathrm{CT}$ and the results of two real time RT-PCR tests taken 24 hours apart were negative for COVID-2019 antigens. 


\section{Statistical analysis}

Baseline demographics and clinical characteristics were compared between asymptomatic infected patients' illness onset or not. Results are presented as mean with standard deviation (SD) or frequency. Pearson's chi-square test or Fisher's exact test was applied to continuous variables or categorical variables. A multivariate Cox regression model was performed to evaluate the potential predictors of COVID-2019 asymptomatic infected patients' illness onset. Variables with $P<0.1$ in the univariate analysis and those closely related to symptom onset were included in the multivariate Cox regression model. All statistical analyses were conducted with SPSS version 25 (International Business Machines Corporation, New York, the Unites States), and $P<0.05$ was considered statistically significant.

\section{Results}

\section{Demographic characteristics}

The study population included 36 males and 34 females and individuals of all ages were involved in the asymptomatic COVID-19 infection with a mean age of 33.24 \pm 20.40 years (ranging from 0.5-84 years). 5 $(5 / 70,7.1 \%)$ of the 70 asymptomatic quarantined patients were associated with familial clusters, 3 $(3 / 70,4.3 \%)$ closely contacted with the confirmed patients, and the rest $(62 / 70,88.6 \%)$ were the exposed population during the tracking of infectious source. 14 cases $(14 / 70,20 \%)$ had coexisting disorders -6 (6/70, $8.6 \%)$ had hypertension, $5(5 / 70,7.1 \%)$ had diabetes, and $3(3 / 70,4.3 \%)$ had pulmonary disease. A total of $11(11 / 70,15.7 \%)$ cases had smoking history, and 3 $(3 / 70,4.3 \%)$ cases had a history of drinking.

\section{Symptoms}

Totally, 22 asymptomatic patients developed symptoms during hospitalization, whereas 48 patients remained asymptomatic throughout the course of the disease. The initial symptoms at onset of illness in 22 patients were fever $(4 / 22,18.2 \%)$, cough $(13 / 22$, $59.1 \%)$, chest discomfort $(2 / 22,9.1 \%)$, fatigue $(1 / 22$, $4.5 \%)$, pharyngalgia $(1 / 22,4.5 \%)$; only one $(1 / 22$, $4.5 \%$ ) patient first developed cough and pharyngalgia at the same time. The mean time from nucleic acid positive to symptoms onset was $4.41 \pm 3.55$ days (ranging from 1-14 days). The details are shown in Figures 1 and 2.

\section{Radiologic and laboratory findings}

Abnormalities on chest CT were detected among 35 of the 69 patients $(35 / 69,50.7 \%)$ after admission, except for one pregnant woman who had no symptoms and not meant examined. The images of 35 patients with positive lung CT were diverse, among which GGO was the most common $(28 / 35,80.0 \%)$, followed by interstitial abnormalities $(3 / 35,8.6 \%)$, local patchy shadowing $(2 / 35,5.7 \%)$ and subpleural shadowing (2/35, 5.7\%). In addition, 15 patients $(15 / 35,50.1 \%)$ had bilateral involvement. The mean time from positive tests with nucleic acid to abnormalities on chest CT was $2.91 \pm 3.35$ days (ranging from 0-16 days). After admission, 4 (4/70, $5.7 \%)$ and $8(8 / 70,11.4 \%)$ cases showed leucopenia (white blood cell count $<3.5 \times 10^{9} / \mathrm{L}$ ) and lymphopenia (lymphocyte count $<1.1 \times 10^{9} / \mathrm{L}$ ). The detailed information of clinical characteristics and laboratory results are listed in Table $\mathbf{1 .}$

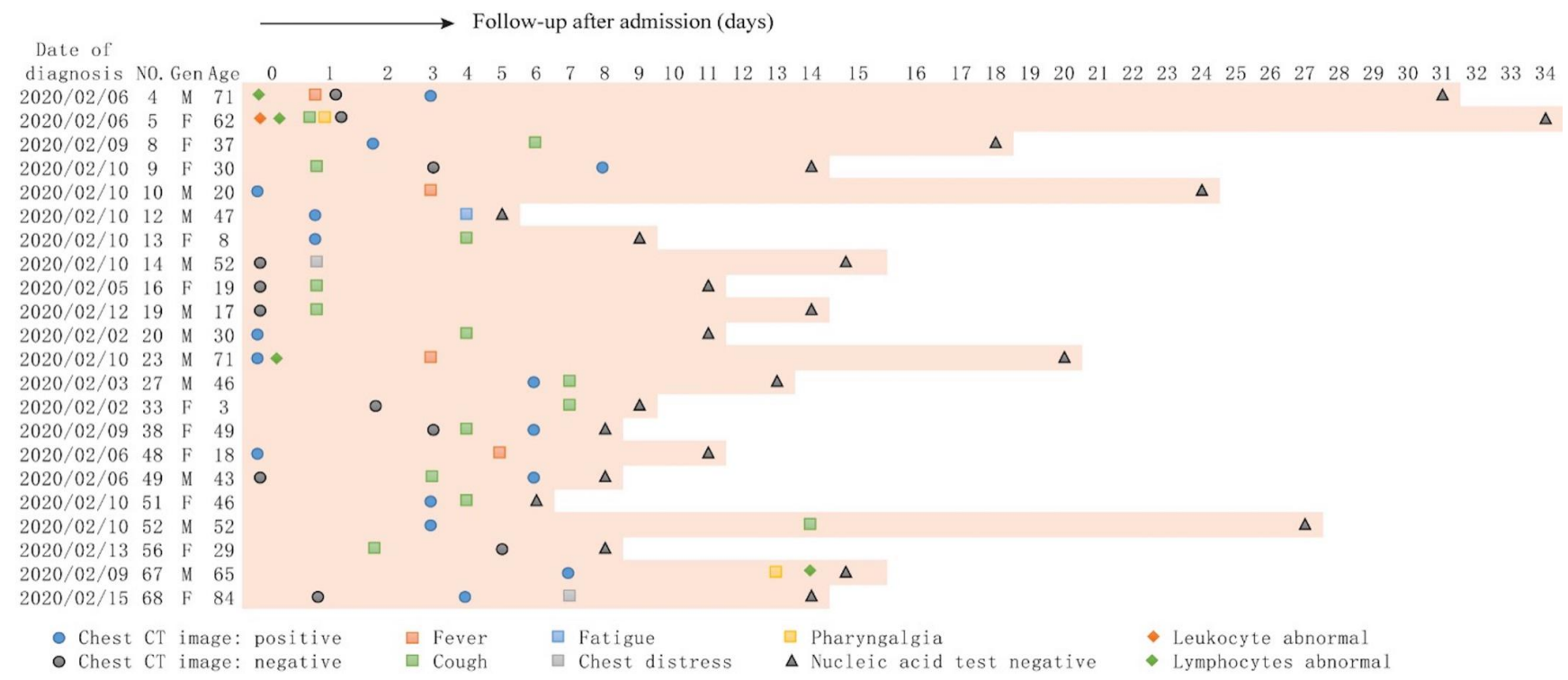

Figure 1. The clinical characteristic of the 22 symptomatic patients. Date of diagnosis (nucleic acid test positive) was defined as origin point, abnormal blood test and chest $\mathrm{CT}$ image upon admission were listed; and symptoms and nucleic acid test were recorded during hospitalization. 
Table 1. Personal and clinical characteristics of 70 asymptomatic COVID-19 infected patients in Hunan province, China

\begin{tabular}{|c|c|c|c|c|}
\hline & $\begin{array}{l}\text { All asymptomatic } \\
\text { patients no. }(\%),(\mathrm{N}=70)\end{array}$ & $\begin{array}{l}\text { Remain asymptomatic } \\
\text { no. }(\%),(\mathrm{N}=48)\end{array}$ & $\begin{array}{l}\text { Symptom onset } \\
\text { no. }(\%),(\mathrm{N}=22)\end{array}$ & $P$ value \\
\hline Age mean (years) & $33.24 \pm 20.40$ & $29.74 \pm 18.92$ & $40.86 \pm 21.85$ & 0.034 \\
\hline Gender & & & & 1 \\
\hline Male & $36(51.4)$ & $25(52.1)$ & $11(50.0)$ & \\
\hline Female & $34(48.6)$ & $23(47.9)$ & $11(50.0)$ & \\
\hline \multicolumn{5}{|l|}{ Coexisting conditions } \\
\hline Hypertension & $6(8.6)$ & $3(6.3)$ & $3(13.6)$ & 0.37 \\
\hline Diabetes & $5(7.1)$ & $3(6.3)$ & $2(9.1)$ & 0.646 \\
\hline Pulmonary disease & $3(4.3)$ & $1(2.1)$ & $2(9.1)$ & 0.231 \\
\hline Drinking history & $3(4.3)$ & 0 & $3(13.6)$ & 0.028 \\
\hline Smoking history & $11(15.7)$ & $4(8.3)$ & $7(31.8)$ & 0.029 \\
\hline \multicolumn{5}{|l|}{ Initial symptoms during quarantine } \\
\hline Fever & $4(5.7)$ & & $4(18.2)$ & \\
\hline Cough & $13(18.6)$ & & $13(59.1)$ & \\
\hline Chest distress & $2(2.9)$ & & $2(9.1)$ & \\
\hline Fatigue & $1(1.4)$ & & $1(4.5)$ & \\
\hline Muscle pain & 0 & & 0 & \\
\hline Pharyngalgia & $1(1.4)$ & & $1(4.5)$ & \\
\hline Cough and pharyngalgia & $1(1.4)$ & & $1(4.5)$ & \\
\hline Time interval from RT-PCT positive to symptom onset mean (days) & & & $4.41 \pm 3.55$ & \\
\hline Chest CT scan abnormal & $35(50.7)$ & $19(40.4)$ & $16(72.7)$ & 0.019 \\
\hline Ground-glass shadow & $28(40.0)$ & $14(29.2)$ & $12(54.5)$ & \\
\hline Interstitial abnormalities & $3(4, .3)$ & $2(4.2)$ & $1(4.5)$ & \\
\hline Local patchy shadowing & $2(2.9)$ & $1(2.1)$ & $1(4.5)$ & \\
\hline Subpleural shadowing & $2(2.9)$ & 0 & $2(18.2)$ & \\
\hline Time from positive chest CT scan to RT-PCR tests mean (days) & $2.91 \pm 3.35$ & $2.74 \pm 3.84$ & $3.13 \pm 2.75$ & 0.732 \\
\hline Time from positive to negative tests with RT-PCR mean (days, range) & $13.20 \pm 6.84(3-34)$ & $12.46 \pm 6.23(3-32)$ & $14.82 \pm 7.94(5-34)$ & 0.18 \\
\hline Leucocytes count mean $\left(\times 10^{9} / \mathrm{L}\right)$ & $6.09 \pm 1.79$ & $6.20 \pm 1.96$ & $5.84 \pm 1.32$ & 0.435 \\
\hline Increased $(>9.5)$ & $4(5.7)$ & $4(8.3)$ & 0 & \\
\hline Normal range (3.5-9.5) & $62(88.6)$ & $41(85.4)$ & $21(95.5)$ & \\
\hline Decreased $(<3.5)$ & $4(5.7)$ & $3(6.3)$ & $1(4.5)$ & \\
\hline Lymphocytes count mean $\left(\times 10^{9} / \mathrm{L}\right)$ & $2.66 \pm 5.97$ & $2.06 \pm 1.02$ & $3.96 \pm 10.59$ & 0.216 \\
\hline Increased $(>3.2)$ & $6(8.6)$ & $5(10.4)$ & $1(4.5)$ & \\
\hline Normal range (1.1-3.2) & $56(80.0)$ & $38(79.2)$ & $18(81.8)$ & \\
\hline Decreased $(<1.1)$ & $8(11.4)$ & $5(10.4)$ & $3(13.6)$ & \\
\hline \multicolumn{5}{|l|}{ Epidemiology } \\
\hline Familial cluster & $5(7.1)$ & $4(8.3)$ & $1(4.5)$ & \\
\hline Close contact history & $3(4.3)$ & $3(6.3)$ & 0 & \\
\hline Exposure history & $62(88.6)$ & $41(85.4)$ & $21(95.5)$ & \\
\hline \multicolumn{5}{|l|}{ Treatment } \\
\hline Interferon atomization & $6(8.6)$ & $2(4.2)$ & $4(18.2)$ & \\
\hline Interferon atomization + Arbidol hydrochloride & $5(7.1)$ & $4(8.3)$ & $1(4.5)$ & \\
\hline Interferon atomization + Oseltamivir & $1(1.4)$ & $1(2.1)$ & 0 & \\
\hline Interferon atomization + Ribavirin & $1(1.4)$ & $1(2.1)$ & 0 & \\
\hline Interferon atomization + Lopinavi & $33(47.1)$ & $22(45.8)$ & $11(50)$ & \\
\hline Interferon atomization + Lopinavir + Arbidol hydrochloride & $8(11.4)$ & $4(8.3)$ & $4(18.2)$ & \\
\hline Interferon atomization + Lopinavir + Ribavirin & $13(18.6)$ & $12(25)$ & $1(4.5)$ & \\
\hline Lopinavi & $2(2.9)$ & $1(2.1)$ & $1(4.5)$ & \\
\hline Lopinavir + Arbidol hydrochloride & $1(1.4)$ & $1(2.1)$ & 0 & \\
\hline \multicolumn{5}{|l|}{ Clinical outcome } \\
\hline Discharged & $70(100)$ & $48(100)$ & $22(100)$ & \\
\hline
\end{tabular}

Note: All values are expressed as the mean \pm standard deviation or number (\%) as appropriate according to the primary studies.

\section{Treatment and clinical outcome}

Antiviral therapy was given to all 70 cases as initiated therapy, among which $6(6 / 70,8.6 \%)$ and 2 patients $(2 / 70,2.9 \%)$ received interferon atomization and lopinavi alone respectively, and the other 62 patients $(72 / 70,88.6 \%)$ received more than 2 antiviral treatments (The details are shown in Table 1). After an effective treatment, none of the cases developed severe pneumonia or admission to intensive care unit, and none died. As of February 18, 2020, all the 70 asymptomatic COVID-19 infected patients had been discharged.

\section{Potential predictors of asymptomatic patients' illness onset}

The mean follow-up time was $9.93 \pm 6.66$ days, ranging from 1-32 days. The mean time from nucleic acid positive to negative was $13.2 \pm 6.84$ days (range, 3-34 days). Age $\left(\chi^{2}=4.51, P=0.036\right)$, pulmonary disease history $\left(\chi^{2}=2.99, P=0.104\right)$, smoking history $\left(\chi^{2}=5.42\right.$, $P=0.026)$, drinking history $\left(\chi^{2}=4.61, P=0.044\right)$, and 
positive chest CT scan $\left(\chi^{2}=4.66, P=0.038\right)$ of asymptomatic carriers were statistically different in the univariate Cox regression model, these factors as well as interested factors, including gender, leukocyte and lymphocyte classification were embraced in the multivariate Cox regression analysis. The results showed that history of smoking $(P=0.028$, hazard ratio $=4.49$, 95\% CI: 1.18-17.08) and pulmonary disease $(P=0.038$, hazard ratio $=7.09,95 \% \quad C I$ : 1.12-44.90) were predictors of the onset of symptoms in asymptomatic carries with COVID-19 (Table 2).

\section{Discussion}

Currently, the number of asymptomatic infected patients with COVID-19 is rampaging around the world and previous evidence has pointed out human to human transmission, which plays a minor role and intensities the difficulty of prevention and management in the epidemic overall. Owing to lack of distinct clinical symptoms for asymptomatic carriers in the initial stage of infection, the disease information may be not provided with full attention. The current study presented clinical characteristics and evaluated the potential factors for symptoms onset in asymptomatic COVID-19 infected patients during quarantined. The results showed that the clinical manifestations and laboratory examinations of asymptomatic patients were nonspecific, and had a good prognosis. History of smoking and pulmonary disease were risk factors of symptoms onset in asymptomatic carriers. Therefore, in order to better predict and control the development of disease, it is necessary to identify and isolate asymptomatic infections at an early stage, and to consider the impact of individuals' heterogeneity on the course of the disease.

There 11 and 3 asymptomatic carriers presented history of smoking and pulmonary disease respectively in the current study. Although the risk factors of COVID-19 remain unclear, previous studies reported that a significant proportion of infected patients had underlying diseases [3,9-12]. Guo L, et al designed a predictive tool using the MuLBSTA score system, and revealed age, hypertension, smoking history, namely multilocular infiltration, lymphopenia, and bacterial co-infection were 6 poor prognostic factors in patients with viral pneumonia [13]. Further investigation is required to explore the applicability of the MuLBSTA score in predicting the risk of COVID-2019 infection. Another study showed the indicators of disease severity associated with poor clinical outcomes, including leukocyte and lymphocyte count, oxygenation, respiratory rate, and chest imaging findings [10]. In summary, previous studies suggested that patients who were older, with underlying diseases, and history of smoking were more likely to increase disease severity and predict a poor outcome [9-12,14]. However, these results need further clarification, since the reported subjects were all confirmed symptomatic COVID-2019 patients, and most of the reported individuals had died or remained hospitalized before the results publication.

The present study showed that initial signs of asymptomatic infected patients were cough, followed by fever. As we all know, the virus particles can spread through the respiratory mucosa, act on $\mathrm{T}$ lymphocytes and induce cytokine storm in the body, give rise to a series of immune responses, and cause changes of immune cells such as peripheral blood leukocytes and lymphocytes. In terms of laboratory tests, most confirmed COVID-2019 patients showed lymphopenia, and normal or decreased leukocyte count $[5,10,13]$. A prospective study of 61 COVID-2019 patients, including 44 mild and 17 moderate to severe patients, found that neutrophil to lymphocyte ratio (NLR) was a reliable early marker of severe progression in COVID-19 patients [14]. However, all of these patients were already showing varying degrees of symptoms at the time of diagnosis and most of them were still hospitalized, with conditions may change during follow-up, and the study has not included the ultimate survival outcomes.

Table 2. The risk factors of COVID-2019 asymptomatic infected patients' illness onset

\begin{tabular}{|c|c|c|c|c|}
\hline & \multicolumn{2}{|c|}{ Univariate analysis } & \multicolumn{2}{|c|}{ Multivariate analysis } \\
\hline & OR $(95 \% \mathrm{CI})$ & $P$-value & $\mathrm{HR}(95 \% \mathrm{CI})$ & $P$-value \\
\hline Age & $1.02(1.00-1.04)$ & 0.036 & $1.01(0.98-1.04)$ & 0.372 \\
\hline Gender (male vs female) & $1.05(0.46-2.43)$ & 0.904 & $0.25(0.06-1.04)$ & 0.056 \\
\hline \multicolumn{5}{|l|}{ Coexisting conditions } \\
\hline Hypertension & $0.49(0.14-1.67)$ & 0.254 & & \\
\hline Diabetes mellitus & $0.67(0.16-2.89)$ & 0.595 & & \\
\hline Pulmonary disease & $0.30(0.07-1.28)$ & 0.104 & $7.09(1.12-44.90)$ & 0.038 \\
\hline Drinking history (presence vs absence) & $0.29(0.08-0.97)$ & 0.044 & $2.50(0.60-10.35)$ & 0.207 \\
\hline Smoking history (presence vs absence) & $0.36(0.14-0.88)$ & 0.026 & $4.49(1.18-17.08)$ & 0.028 \\
\hline Chest CT scan positive (presence vs absence) & $0.37(0.14-0.95)$ & 0.038 & $1.66(0.50-5.51)$ & 0.411 \\
\hline Leucocytes count (decreased vs without decreased) & $1.20(0.16-8.93)$ & 0.858 & $0.59(0.06-5.31)$ & 0.637 \\
\hline Lymphocytes count (decreased vs without decreased) & $0.81(0.24-2.75)$ & 0.742 & $1.79(0.37-8.69)$ & 0.473 \\
\hline
\end{tabular}




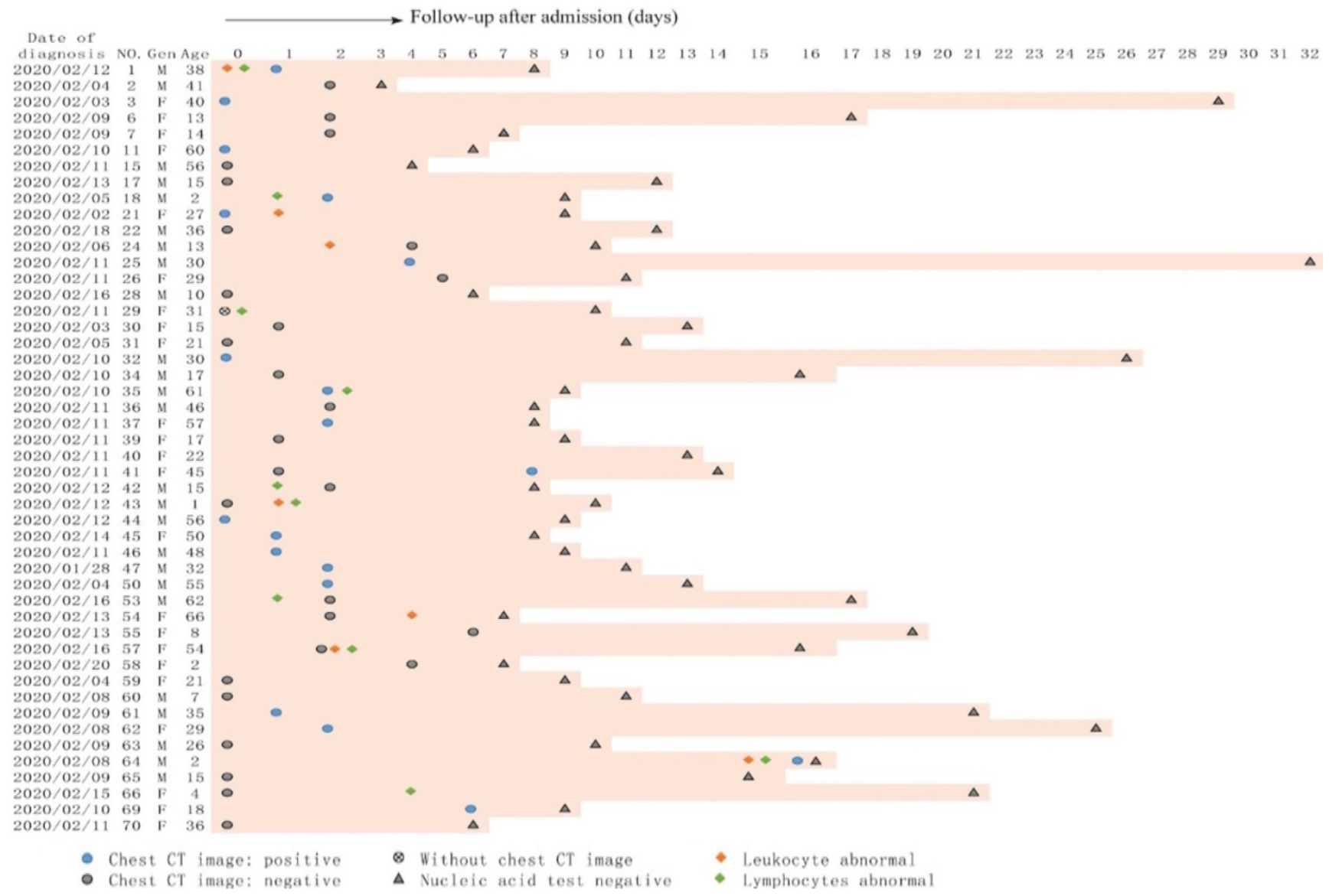

Figure 2. The clinical characteristic of the 48 patients who remained asymptomatic during hospitalization. Date of diagnosis (nucleic acid test positive) was defined as origin point, abnormal blood test and chest CT image upon admission were listed; and nucleic acid test were recorded during hospitalization.

Indeed, the chest CT imaging is important for screening asymptomatic COVID-19 patients and aiding quarantine and infection control strategies. However, due to differences among individual and disease severity, the onset of symptoms do not necessarily present with chest CT abnormalities, as previous study described [15]. Negative of chest CT imaging finding may reflect that the disease is still in an early stage and repeated subsequent CT scan is always needed to determine changes of disease. However, current study showed that chest CT findings negative and laboratory tests were not risk factors for symptomatic attack in asymptomatic patients, which may be associated with the exposure time of patients, individual immune status, and improvement in the patient's condition after rapid treatment during hospitalization. Therefore, the asymptomatic infection patients should be screened and sampled at the same time, nucleic acid detection and chest CT scans should be carried out as early as possible. Moreover, growing evidences are showing that lung ultrasound may be considered a useful alternative to chest $\mathrm{CT}$ for diagnosis and management of COVID-19 given its no X-ray exposure, bedside application, reduced exposition between healthy and infected patients, and was repeatable for follow-up, particularly in pregnant women [16-18].

After an effective antiviral treatment, all the 70 asymptomatic infections had been discharged. The common characteristics of those asymptomatic carriers tended to be younger, with a mean age of $33.24 \pm 20.40$ years, and the mean age was markedly higher in cases of developed symptoms than remained asymptomatic cases $(40.86 \pm 21.85$ vs $29.74 \pm 18.92)$. The result may be attributed to the following: (a) due to individual differences such as age and physical condition, for asymptomatic carriers with strong immunity and physical fitness, particularly who remained asymptomatic throughout the course of disease, there is a certain degree of infectivity but may be less pathogenicity, or body's immune system can kill the virus; (b) for those who are still in the incubation period without symptoms, the human body and the virus may have reached a kind of new symbiotic state. The virus can multiply in the body and spread to the outside world, and become an important source of infection. The combination of prevention and control in China has enabled the asymptomatic infections to receive timely treatment, early isolation, examination and treatment, 
which contain the progress of the disease effectively.

There are several limitations in the present study. First, due to the retrospective nature, only 70 asymptomatic carriers were included during the study period. However, these estimates will be refined as the number of affected asymptomatic carriers is rampaging and being collected. Second, as the patients were only from Hunan province, it would be better to include as many asymptomatic carriers as possible in other cities in China, and even other countries to get a more comprehensive understanding of asymptomatic carriers with COVID-2019. Further validation is needed in larger multi-institutional studies. Third, lung ultrasound can be used to monitor lung involvement during a specific treatment and can be easily used in every setting, as previous study described, but we lack of lung ultrasound studies. Lastly, the date of nucleic acid test positive to negative may be shorter than the 10-day hospitalization, which could result in biases of clinical observation features.

In conclusion, COVID-19 can present as asymptomatic carriage. The clinical manifestations of initially asymptomatic patients presented mild symptoms and a good prognosis. Histories of smoking and pulmonary disease were prone to illness onset in asymptomatic infected patients with COVID-19, and more attention should be paid for those individuals. Further studies are needed to elucidate the clinical characteristics, risk and prognostic factors of asymptomatic carriers.

\section{Abbreviations}

COVID-2019: coronavirus disease 2019; SARSCoV-2: severe acute respiratory syndrome corona virus 2; WHO: World Health Organization; RT-PCR: reverse transcription polymerase chain reaction; $\mathrm{CT}$ : computed tomography; CIOMS: Council for International Organization of Medical Sciences; GGO: ground-glass opacity; WBC: leukocyte count; LY: lymphocyte count; NUET: neutrophil ratio; LYM: lymphocyte ratio; SD: standard deviation; NLR: neutrophil to lymphocyte ratio.

\section{Acknowledgments}

\section{Funding}

Key Emergency Project of Pneumonia Epidemic of novel coronavirus infection (2020SK3006). Emergency Project of Prevention and Control for COVID-19 of Central South University (160260005). The funders of the study had no role in study design, data collection, data analysis, data interpretation, or writing of the report. We thank all patients involved in the study.

\section{Author Contributions}

Yu-Dong Xiao and Ling Leng conceptualized the project. Kun Liu, Ri-Hua Zhou and Yue-Chun $\mathrm{Hu}$ collected the epidemiological, laboratory and clinical data. Pei-Yao Tao and Ling Leng performed statistical analysis. Pei-Yao Tao contributed to writing of the report. Yu-Dong Xiao, Jun Liu, and Shang-Jie $\mathrm{Wu}$ contributed to critical revision of the report. All authors had full access to all data in the study, take responsibility for the integrity of the data and the accuracy of the data analysis, and reviewed and approved the final version.

\section{Competing Interests}

The authors have declared that no competing interest exists.

\section{References}

1. Wang C, Horby PW, Hayden FG, et al. A novel coronavirus outbreak of global health concern. Lancet. 2020; 395 (10223): 470-3.

2. Liu YC, Liao CH, Chang CF, et al. A Locally Transmitted Case of SARS-CoV-2 Infection in Taiwan. N Engl J Med. 2020; 382 (11): 1070-2.

3. The Novel Coronavirus Pneumonia Emergency Response Epidemiology Team. Vital Surveillances: The Epidemiological Characteristics of an Outbreak of 2019 Novel Coronavirus Diseases (COVID-19) - China, 2020. China CDC Weekly. 2020; 2(8): 113-2.

4. Bai Y, Yao L, Wei T, et al. Presumed Asymptomatic Carrier Transmission of COVID-19. JAMA. 2020; 323 (14): 1406-7.

5. China National Health Commission. Diagnosis and treatment of pneumonitis caused by new coronavirus (trial version 6). Beijing: China National Health Commission.

http://www.nhc.gov.cn/yzygj/s7653p/202001/4294563ed35b43209b31739bd 0785e67.shtml.

6. Rothe C, Schunk M, Sothmann P, et al. Transmission of 2019-nCoV Infection from an Asymptomatic Contact in Germany. N Engl J Med. 2020; 382 (10): 970-1.

7. Yu P, Zhu J, Zhang Z, et al. A familial cluster of infection associated with the 2019 novel coronavirus indicating potential person-to-person transmission during the incubation period. J. Infect. Dis. 2020; 221 (11): 1757-1761.

8. Zou L, Ruan F, Huang M, et al. SARS-CoV-2 Viral Load in Upper Respiratory Specimens of Infected Patients. N Engl J Med. 2020; 382 (12): 1177-9.

9. Guan WJ, Ni ZY, Hu Y, et al. Clinical Characteristics of Coronavirus Disease 2019 in China. N Engl J Med. 2020; 382: 1708-1720.

10. Chen N, Zhou M, Dong X, et al. Epidemiological and clinical characteristics of 99 cases of 2019 novel coronavirus pneumonia in Wuhan, China: a descriptive study. Lancet. 2020; 395 (10223): 507-13.

11. Huang C, Wang Y, Li X, et al. Clinical features of patients infected with 2019 novel coronavirus in Wuhan, China. Lancet. 2020; 395 (10223): 497-506.

12. Wang D, Hu B, Hu C, et al. Clinical Characteristics of 138 Hospitalized Patients With 2019 Novel Coronavirus-Infected Pneumonia in Wuhan, China. JAMA. 2020.

13. Guo L, Wei D, Zhang X, et al. Clinical Features Predicting Mortality Risk in Patients With Viral Pneumonia: The MuLBSTA Score. Front Microbiol. 2019; 10: 2752.

14. Liu JY, Liu Y, Xiang P, et al. Neutrophil-to-lymphocyte ratio predicts critical illness patients with 2019 coronavirus disease in the early stage. J Transl Med. 2020; 18 (1): 206

15. Zu ZY, Jiang MD, Xu PP, et al. Coronavirus Disease 2019 (COVID-19): A Perspective from China. Radiology. 2020; 296: E15-E25.

16. Musolino AM, Supino MC, Buonsenso D, et al. Lung Ultrasound in Children with COVID-19: Preliminary Findings. Ultrasound Med Biol. 2020; 46 (8): 2094-2098.

17. Buonsenso D, Raffaelli F, Tamburrini E, et al. Clinical role of lung ultrasound for diagnosis and monitoring of COVID-19 pneumonia in pregnant women. Ultrasound Obstet Gynecol. 2020; 56 (1): 106-109.

18. Buonsenso D, Pata D, Chiaretti A. COVID-19 outbreak: less stethoscope, more ultrasound. Lancet Respir Med. 2020; 8 (5): e27. 